Int. J. Morphol.,

33(3):996-1001, 2015.

\title{
Índices de Proporcionalidad y Composición Corporal de la Élite de Gimnasia Acrobática
}

\author{
Proportionality Indices and Body Composition of Elite Acrobatic Gymnasts
}

Yaiza Taboada-Iglesias*; Águeda Gutiérrez-Sánchez* \& Mercedes Vernetta***

TOBOADA-IGLESIAS Y.; GUTIÉRREZ-SÁNCHEZ, Á. \& VERNETTA, M. Índices de proporcionalidad y composición corporal de la élite de gimnasia acrobática. Int. J. Morphol., 33(3):996-1001, 2015.

RESUMEN: El objetivo del estudio fue determinar los índices de proporcionalidad y la composición corporal de la Gimnasia Acrobática estableciéndose una diferenciación en función del rol. La muestra fue compuesta por 150 gimnastas de toda España. Se realizó el análisis y la comparación de los diferentes índices de proporcionalidad y la composición corporal en ambos grupos (ágiles y portores). El porcentaje de grasa fue significativamente $(\mathrm{p}<0,001)$ superior en los portores $(15,1 \%$ de grasa), mientras que el porcentaje óseo fue mayor en los ágiles $(19,82 \%)$. En cuanto a los índices de proporcionalidad no se encontraron diferencias significativas entre ambos roles. Se aprecian diferencias significativas en relación al porcentaje de grasa y óseo entre los ágiles y los portores, siendo el porcentaje graso inferior en los ágiles y el óseo inferior en los portores. Ambos grupos se caracterizan por tener extremidades superiores cortas, siendo las inferiores largas en los ágiles e intermedias en los portores. Ambos roles presentan un tronco intermedio y de forma intermedia.

PALABRAS CLAVE: Antropometría; Composición corporal; Morfología; Gimnasia acrobática; Gimnasia.

\section{INTRODUCCIÓN}

La determinación de los parámetros antropométricos de las diferentes especialidades deportivas es una de las líneas que sigue las Ciencias del Deporte persiguiendo diferentes objetivos. Establecer un perfil de alto rendimiento específico para cada deporte es uno de ellos para poder realizar una buena detección de talentos y alcanzar el mayor rendimiento posible (Claessens et al., 1999; Di Cagno et al., 2009; Slezynski \& Swiat, 1997; Rodríguez et al., 2014). Dentro de la especialización en cada deporte también es necesario determinar patrones morfológicos más aptos para un determinado puesto o rol (Krüger et al., 2014; Slezynski \& Swiat).

Por otro lado, Classens et al. (1996), establecieron que el perfil morfológico en gimnasia se encuentra relacionado con la tasa de lesión, por lo que los estudios antropométricos permiten realizar una mejor prevención de lesiones.

La composición corporal y los índices de proporcionalidad son parámetros muy estudiados en ciertas disciplinas gimnásticas pertenecientes a la Federación Internacional de Gimnasia (FIG) como la Gimnasia Artística Masculina y Femenina (GAM y GAF), Gimnasia Rítmica (GR), Trampolín y Gimnasia Aeróbica (GAer) donde existe una mayor evidencia científica referente a la tipología morfológica (Claessens et al., 1991, 1999; Bester \& Coetzee, 2010; Díaz et al., 2008; Ávila-Carvalho et al., 2012; Klentrou \& Plyley, 2003; Lopez-Benedicto et al., 1991; Quintero et al., 2011; Vernetta et al., 2011; Gómez-Landero et al., 2004, 2009; López Bedoya et al., 1999; Mineva, 2011)

No obstante en Gimnasia Acrobática (GA), al ser la última disciplina gimnástica integrada en la FIG en 1999, los estudios se encuentran en las primeras etapas de desarrollo, por lo que no existen muchos trabajos científicos, siendo el de Slezynski \& Swiat uno de los realizados sobre el perfil antropométrico de gimnastas de GA de clase mundial y gimnastas polacos.

La GA es una disciplina que está incrementando tanto el nivel como en participación. Se trata de un deporte sociomotriz, donde existe siempre la presencia de uno o varios compañeros que sincronizan sus acciones en un espacio estable reglado $(12 \times 12 \mathrm{~m})$, para conseguir la realización de lanzamientos, figuras y pirámides humanas (Vernetta et al., 2008). La combinación de elementos individuales y grupales sincronizados a un soporte musical, constituye la

\footnotetext{
" Departamento de Didácticas Especiales. Facultad de Ciencias de la Educación y del Deporte, Grupo GiES10, Instituto de Investigación Biomédica (IBI) Universidad de Vigo, Pontevedra, España.

** Departamento de Educación Física y Deporte. Facultad de Ciencias del Deporte. Universidad de Granada, Granada, España.
} 
esencia de esta disciplina. Los gimnastas compiten en parejas (masculinas, femeninas o mixtas) o grupos (tríos femeninos o cuartetos masculinos).

Para la realización de los elementos grupales se diferencian los roles de portores o ágiles siendo desempeñados ambos roles por distintos sexos. El portor es la persona que sujeta y el ágil la persona que realiza los elementos de flexibilidad, equilibrio y combinaciones encima del portor o, grandes saltos acrobáticos en fase aérea mediante propulsiones de los portores para recepcionar de nuevo sobre ellos o en el suelo (Vernetta et al., 2007). El desempeño de un determinado rol adquiere mayor importancia que el sexo del gimnasta, así como confirmó un estudio realizado en Gimnasia Rítmica por Di Cagno et al. (2009) que sugiere que los hombres y las mujeres con mayor nivel no presentan diferencias antropométricas.

Las características físicas y morfológicas individuales de los gimnastas influencian en gran medida la ejecución de estos elementos grupales, siendo difícil substituir a un miembro del equipo con poco tiempo de antelación. Por este motivo, las lesiones de un solo gimnasta conllevan la retirada de todo el grupo de la competición (Purnell et al., 2010).

El objetivo del estudio fue determinar los índices de proporcionalidad y la composición corporal de esta modalidad gimnástica estableciéndose una diferenciación en función al rol que desempeñan.

\section{MATERIAL Y MÉTODO}

Se evaluaron 150 gimnastas participantes en el Campeonato de España de 2013 con una media (X) de edad de 13,31 y desviación típica (DT) de 3,1, siendo éstos la totalidad de los gimnastas españoles de nivel nacional e internacional, así como los integrantes de la Selección Nacional de GA. Se agruparon en función del rol que desempeñan en su actuación, siendo 58 ágiles con una media de edad de 11,30 años $(\mathrm{DT}=2,97)$ y 92 portores con una media de 14,58 años (DT= $2,67)$. No se ha tenido en cuenta la diferenciación dicotómica de sexo, ya que la lógica interna de esta modalidad gimnástica establece que ambos roles son desempeñados por ambos sexos indistintamente.

La investigación cumplió los preceptos de la Declaración de Helsinki. Todos los sujetos participaron de forma voluntaria y dado el tipo de estudio y las técnicas utilizadas en el mismo, esta investigación respetó todos los procedimientos éticos para la recogida de datos y la Ley Orgánica 15/1999, sobre protección de datos de carácter personal. Los padres de los sujetos menores firmaron el consentimiento informado necesario para efectuar las mediciones.

Las mediciones fueron tomadas en las instalaciones deportivas de cada club por el mismo investigador, acreditado por la Sociedad Internacional por el avance de la Cineantropometría (ISAK). Se utilizaron los instrumentos aconsejados por la ISAK y todos fueron calibrados antes de su uso.

Las medidas cinenatropométricas registradas fueron peso con una báscula digital Tanita con precisión de $100 \mathrm{~g}$, talla (T) y talla sentado (TS) mediante un estadiómetro portátil, envergadura, 4 pliegues cutáneos (tríceps, subescapular, ileocrestal y abdominal) medidos con un plicómetro Holtain con precisión de 0,2 $\mathrm{mm}$, cuatro diámetros (biacromial, biileocrestal, bicondíleo del fémur y biestiloideo de muñeca) mediante un calibre para pequeños diámetros Holtain con precisión de $1 \mathrm{~mm}$ y antropómetro Harpenden (Holtain) y la longitud del miembro superior (LMS) antropómetro Harpenden (Holtain). Se realizaron las mediciones controlando en todo momento un error técnico de medida no superior al $5 \%$ en los pliegues y el $2 \%$ en el resto de medidas.

A partir de estas mediciones se han calculado diferentes índices y parámetros. La longitud de los miembros inferiores (LMI) siendo la diferencia entre talla y talla sentado. Índices de proporcionalidad; índice ponderal (IP) (peso/estatura3), longitud relativa de los miembros superiores (LRMS) siendo ésta la [(LMS/T)x100], longitud relativa de los miembros inferiores (LRMI) calculada como (LMI/T)x100, Índice córmico (IC) calculado [(TS/T)x100], índice esquelético (IE) siendo éste ([(T-TS)/ TS]x100), índice acromio-ilíaco (IAI) [(diámetro biileocrestal / diámetro biacromial)x100] y envergadura relativa (ER) [(envergadura/T)x100]. Se ha seguido la estrategia de De Rose \& Guimaraes (1980) basada en el método de cuatro componentes propuesto por Matiegka (1921) para establecer la composición corporal calculada mediante la fórmula de Faulkner para el porcentaje de grasa, el porcentaje óseo por la fórmula de Von Doblen modificada por Rocha, peso residual a partir de la fórmula de Wurch y porcentaje de masa muscular a partir de los valores anteriores.

Se utilizó una hoja de cálculo elaborada por el Grupo Español de Cineantropometría (CREC) de la Federación Española de Medicina Deportiva (FEMEDE) en 2008 para la recogida de datos.

El análisis estadístico fue realizado con el programa SPSS 15.0 (Statistical Package for the Social Sciences). Se analizaron como medida de tendencia central la media y como medidas de dispersión se ha utilizado la desviación típica (DT). Se han incluido además los valores máximos y mínimos (rango) de cada variable. Los datos han sido sometidos a la prue- 
ba Z de Kolmogorov-Smirnov para comprobar la normalidad. Para el análisis comparativo se realizó la prueba T student para comparar medidas independientes y la U de Mann-Whitney para las variables que no mantenían una distribución normal u homogénea.

\section{RESULTADOS}

En la Tabla I se muestra la composición corporal y los índices de proporcionalidad de ambos roles y su análisis comparativo.

Todas las variables presentan una distribución normal ( $p<0,05)$ excepto LRMS, ER y el porcentaje residual, por lo que a éstas se les pasó la prueba no paramétrica $U$ de Man-Whitney para la comparación en función del rol.

La prueba de Levene indica que existe homogeneidad de varianzas en las variables, excepto porcentaje de grasa $(\mathrm{F}<0,05)$.

El grupo de ágiles presentan una LRMS que establece un morfotipo braquibraquial, referido a miembros superiores cortos. Por otro lado el IC indica que pertenecen al grupo de los mesocórmicos, es decir, tronco medio. El IE indica que los sujetos se denominan macroesqueléticos, miembros inferiores largos. Según el IAI se considera que los ágiles tienen un tronco intermedio y la ER indica que su envergadura es ligeramente inferior a la estatura.

Por otro lado, en el grupo de los portores la LRMS establece un morfotipo braquibraquial, es decir, miembros superiores cortos. El IC indica que pertenecen al grupo de los mesocórmicos, es decir, tronco medio. El IE indica que los sujetos se denominan mesoesquelético, referido a miembros inferiores intermedios. Por otro lado, según el IAI se considera que los portores tienen un tronco intermedio, y la ER indica que la envergadura es ligeramente superior a la estatura.

Se aprecian diferencias significativas $(\mathrm{p}<0,05)$ entre ágiles y portores, en el porcentaje de grasa y en el porcentaje óseo, sin diferencias en el resto de las variables estudiadas. Sin embargo, son mayores las medidas de los portores en todas las variables, menos en el porcentaje muscular y porcentaje óseo.

En cuanto a la composición corporal, el porcentaje de grasa es significativamente superior en los portores, mientras que el porcentaje óseo es mayor en los ágiles.

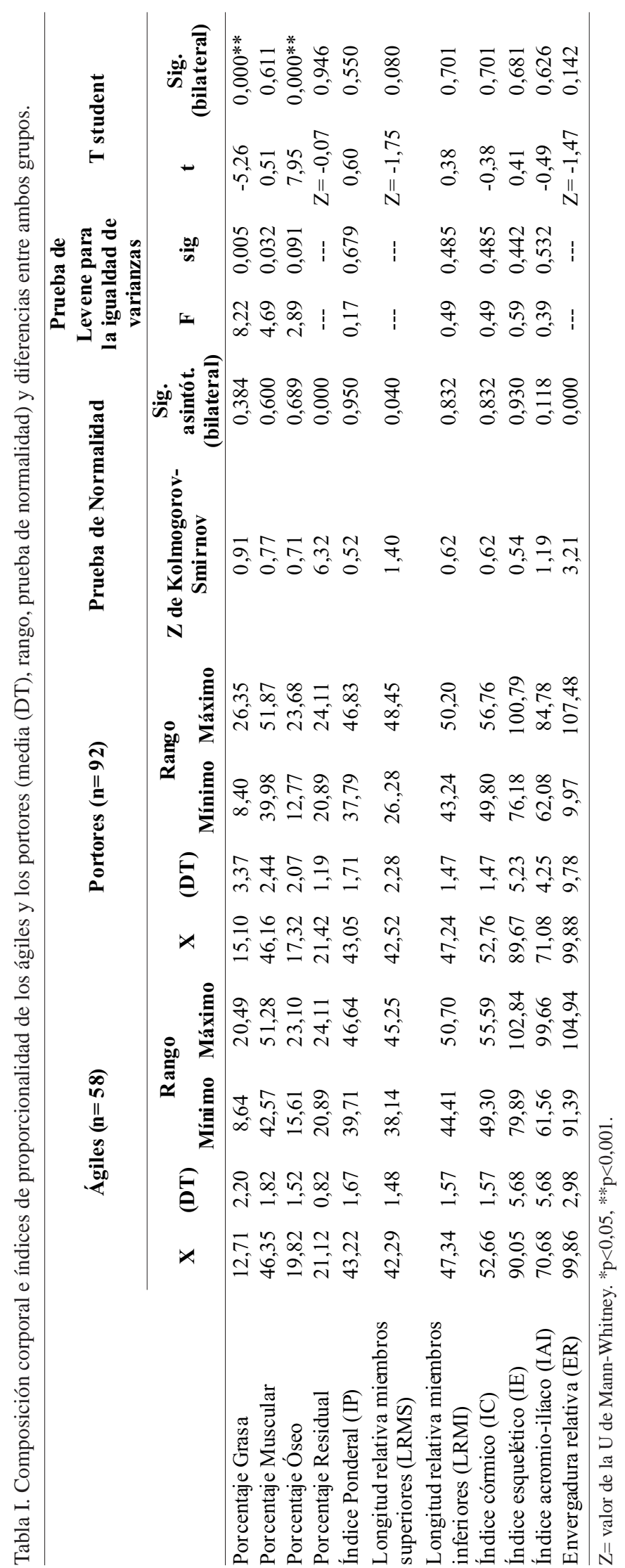




\section{DISCUSIÓN}

Los resultados muestran diferencias significativas en cuanto a la composición corporal, por lo que se afirma que existe una morfología diferenciada en función del rol que desempeña el gimnasta. De ahí que el análisis de la composición corporal tenga gran importancia en el proceso de detección de talentos deportivos (Claessens et al., 1991, 1999; Di Cagno et al., 2008; Slezynski \& Swiat).

Un estudio de Canda et al. (1993) establece diferencias entre las diferentes fórmulas de estimación de la composición corporal, por lo que tan solo se ha discutido con la bibliografía que utiliza la misma metodología que este estudio.

Al comparar los datos con los encontrados en la literatura científica referente a otras modalidades gimnásticas, se aprecian ciertas diferencias y similitudes. Destacar algunos estudios que sugieren que las gimnastas de GAF con más grasa subcutánea tienen puntuaciones más bajas de rendimiento (Claessens et al., 1991, 1999). Bester \& Coetzee en un estudio realizado en GAF, con especialistas en suelo, concluye que las gimnastas se caracterizan por presentar extremidades largas, solo presentes en las extremidades inferiores de los ágiles de nuestro estudio.

Díaz et al., concluyen que las gimnastas de la selección venezolana de GAF presentan unas extremidades inferiores cortas. En cuanto a la longitud del tronco, estas gimnastas presentan un tronco medio al igual que los gimnastas de este estudio.

Las gimnastas de GR con mayor nivel deportivo presentan los porcentajes de grasa más bajos (Ávila-Carvalho et al.; Klentrou \& Plyley). Por otro lado, Lopez-Benedicto et al., establecen que el porcentaje de grasa de las gimnastas de rítmica es de 14,9 (DE 1,05) siendo superior al de los ágiles pero inferior al de los portores. Quintero et al., establecen que todas las categorías de edad de GR presentan valores inferiores al $12,39 \%$ de grasa, inferior tanto al de los ágiles como al de lo portores de nuestro estudio, siendo el porcentaje muscular superior en las gimnasta de rítmica.

Un estudio de Vernetta et al. (2011) establece que en GR se presenta bajo porcentaje de grasa $(9,18 \%)$ y los índices corporales analizados, revelan que estas gimnastas son braquicórmicas o de tronco corto $(55,80)$, macroesquélicas o de miembros largos $(88,22)$ y que tienen tronco trapezoidal. Presentan también un porcentaje graso inferior a los gimnastas de GA y con miembros proporcionalmente más largos a excepción de los miembros inferiores de nuestros ágiles.
Analizando el IC, los gimnasta de GA examinados presentan un tronco de dimensiones medias siendo el tronco de las gimnastas de GR corto (Di Cagno et al., 2008, 2009).

Gómez-Landero et al. (2009), establecen que las gimnastas de Trampolín se caracterizan por tener miembros superiores cortos e inferiores largos al igual que los ágiles de nuestro estudio, mientras que los portores aun teniendo los miembros superiores cortos, presentan inferiores intermedios. Los gimnastas de la selección española masculina de Trampolín estudiados por Gómez-Landero et al. (2004) presentan un porcentaje graso inferior y muscular superior a los gimnastas de GA de este estudio. El IC indica que presentan un tronco largo, el IE muestra que presentan miembros inferiores intermedios como los portores y el IAI muestra un tronco trapezoidal. Las trampolinistas femeninas, muestran valores de porcentaje graso inferiores a los portores de nuestro estudio, y en el caso de las trampolinistas sub-15 también inferiores a los ágiles. En cuanto al porcentaje muscular las trampolinistas presentan valores superiores a los gimnastas de acrobática, no obstante, los ágiles de éste estudio presentan un porcentaje óseo superior (Gómez-Landero et al., 2004).

Los deportistas de GAer se caracterizan por presentar bajos niveles de masa grasa (Mineva). En un estudio realizado en gimnastas de GAer españoles se establece que presentan un porcentaje graso de 7,75\% (DT 1,96) los hombres y un 10,99\% (DT 3,43) las mujeres (López-Benedicto et al.), siendo valores inferiores al porcentaje de grasa de los portores, y más parecido al de los ágiles.

Concluimos que los portores presentan mayores valores en todas las variables estudiadas a excepción del porcentaje óseo y muscular, no obstante solo se aprecian diferencias significativas en relación al porcentaje de grasa y óseo. El grupo de los ágiles se caracteriza por tener miembros superiores cortos e inferiores largos, y un tronco medio y de forma intermedia, mientras que los portores se caracterizan por tener miembros superiores cortos e inferiores intermedios, longitud del tronco medio y forma intermedia.

Se encuentran similitudes en cuanto al bajo porcentaje graso del resto de las disciplinas gimnasticas con los ágiles de GA. En cuanto a la proporcionalidad también se encuentran similitudes en relación a los miembros inferiores largos de los ágiles con los gimnastas de GR y trampolín.

Este trabajo pretende sentar las bases para futuras investigaciones. Cabría la posibilidad de comprobar si las diferentes modalidades de GA presentan diferencias entre ellas, se podrían incluir variables de rendimiento y establecer qué perfiles son los más adecuados para alcanzar el éxito deportivo. 
AGRADECIMIENTOS. Los autores agradecen a todos los gimnastas y entrenadores por su participación.

TOBOADA-IGLESIAS Y.; GUTIÉRREZ-SÁNCHEZ, Á. \& VERNETTA, M. Proportionality indices and body composition of elite acrobatic gymnasts. Int. J. Morphol., 33(3):996-1001, 2015.

SUMMARY: The study is aimed at determining the proportionality indices and body composition in Acrobatic Gymnastics, establishing a differentiation according to the role to be performed. The sample consisted of 150 gymnasts from throughout Spain. Several proportionality indices and the body composition were analyzed in both groups (tops and bases). A comparative analysis between groups was also carried out. The percentage of body fat was significantly $(p<0.001)$ higher in the bases ( $15.1 \%$ fat), whereas the percentage of bone mass was higher in the tops (19.82\%). Regarding the proportionality indices, there were no significant differences between the two roles. However, significant differences were observed with respect to the percentage of body fat and bone mass between the tops and bases, the percentage of body fat being lower in the tops and that of bone mass being lower in the bases. Tops and bases are characterized by short upper limbs; however, tops present long lower limbs, whereas bases have intermediate lower limbs. Both roles present an intermediate trunk, of intermediate shape.

KEY WORDS: Anthropometrics; Body composition; Morphology; Acrobatic gymnastics; Gymnastics.

\section{REFERENCIAS BIBLIOGRÁFICAS}

Ávila-Carvalho, L.; Klentrou, P.; Luz Palomero, M. \& Lebre, E. Body composition profile of elite group rhythmic gymnasts. Sci. Gymnast. J., 4(1):21-32, 2012.

Bester, A. \& Coetzee, B. Die antropometriese vloeritemprestasiedeterminante Van Jong dogtergimnaste. S. Afr. J. Res. Sport Phys. Educ. Recreat., 32(2):13-30, 2010.

Canda, A.; Martin, M. P. \& Rubio, S. Composición corporal según diferentes métodos antropométricos: un estudio en gimnastas de elite. Arch. Med. Deporte, 10(37):11-7, 1993.

Claessens, A. L.; Veer, F. M.; Lefevre, J.; Maes, H.; Steens, G. \& Beunen, G. Anthropometric characteristics of outstanding male and female gymnasts. J. Sports Sci., 9(1):53-74, 1991.

Claessens, A. L.; Lefevre, J.; Beunen, G.; De Smet, L. \& Veer, A. M. Physique as a risk factor for ulnar variance in elite female gymnasts. Med. Sci. Sports Exerc., 28(5):560-9, 1996.

Claessens, A. L.; Lefevre, J.; Beunen, G. \& Malina, R. M. The contribution of anthropometric characteristics to performance scores in elite female gymnasts. J. Sports Med. Phys. Fitness, 39(4):355-60, 1999.
De Rose, E. H. \& Guimaraes, A. G. S. A Model for Optimization of Somatotype in Young athletes. En: Ostyn, M.; Beunen, G. \& Simons, J. (Eds.). Kinanthropometry II. Baltimore, University Park Press, 1980.

Díaz, M.; Mauri, E.; García, Y. \& Jiménez, C. Perfil antropométrico comparativo de la selección nacional de gimnasia artística femenina (2008) y el perfil del campeonato mundial en Rótterdam, Holanda (1987). E. F. Deportes.com Rev. Digit., 13(123), 2008.

Di Cagno, A.; Baldari, C.; Battaglia, C.; Brasili, P.; Merni, F.; Piazza, M.; Toselli, S.; Ventrella, A. R. \& Guidetti, L. Leaping ability and body composition in rhythmic gymnasts for talent identification. J. Sports Med. Phys. Fitness, 48(3):341-6, 2008.

Di Cagno, A.; Baldari, C.; Battaglia, C.; Monteiro, M. D.; Pappalardo, A.; Piazza, M. \& Guidetti, L. Factors influencing performance of competitive and amateur rhythmic gymnastics--gender differences. J. Sci. Med. Sport, 12(3):411-6, 2009.

Gómez-Landero, A.; López Bedoya, J.; Vernetta, M. \& Fernández, E. Análisis de las características morfológicas de la selección española masculina de trampolín. E. F. Deportes.com Rev. Digit., 10(74), 2004.

Gómez-Landero, L. A.; Vernetta, M. \& Bedoya, J. L. Somatotipo y composición corporal en trampolinistas españolas de alto nivel. Arch. Med. Deporte, 26(130):105-17, 2009.

Klentrou, P. \& Plyley, M. Onset of puberty, menstrual frequency, and body fat in elite rhythmic gymnasts compared with normal controls. Br. J. Sports Med, 37(6):490-4, 2003.

Krüger, K.; Pilat, C.; Ueckert, K.; Frech, T. \& Mooren, F. C. Physical performance profile of handball players is related to playing position and playing class. J. Strength Cond. Res., 28(1):117-25, 2014.

López Bedoya, J.; Vernetta, M. \& de la Cruz, J. C. Características morfológicas y funcionales del aeróbic deportivo. Apunt., 55:60-65,1999.

López-Benedicto, A.; Franco-Bonafonte, L. \& Terreros-Blanco, J. L. Gimnasia rítmica. Evolución fisiológica y antropométrica en una temporada. Arch. Med. Deporte, 8(30):127-33, 1991.

Matiegka, J. The testing of physical efficiency. Am. J. Phys. Anthropol., 4(3):223-30, 1921.

Mineva, M. Body mass composition of aerobic gymnastics competitors. Res. Kinesiol., 39(2):231-3, 2011.

Purnell, M.; Shirley, D.; Nicholson, L. \& Adams, R. Acrobatic gymnastics injury: occurrence, site and training risk factors. Phys. Ther. Sport, 11(2):40-6, 2010. 
Quintero, B. R.; Martín, A. P. \& Henríquez, J. J. G. El perfil antropométrico de la gimnasia rítmica. Apunt. Educ. Fis. Deportes, 103(1):48-55, 2011.

Rodríguez, R. F. J.; González Fuenzalida, H. I.; Cordero Ortiz, J. L.; Lagos Nieto, S.; Aguilera Tapia, R. A. \& Barraza Gómez, F. O. Muscle mass estimation and comparison by segment, in young Chilean athletes. Int. J. Morphol., 32(2):703-8, 2014

Slezynski, J. \& Swiat, T. Budowa somatyczna zawodnikow w akrobatyce sportowej. Wychowanie Fizyczne i Sport, 41(4):s3960, 1997.

Vernetta, M.; López Bedoya \& Gutiérrez, A. La Creatividad en la Gimnasia Acrobática. En: Martínez, A. \& Díaz, P. (Coord.). Creatividad y Deporte: consideraciones teóricas e investigaciones breves. Sevilla, Wanceulen, 2008. pp.133-54.

Vernetta, M.; Fernández, E.; López-Bedoya, J.; Gómez- Landero, A. \& Oña, A. Estudio relacional entre el perfil morfológico y estima corporal en la selección andaluza de gimnasia rítmica deportiva. Mot. Eur. J. Hum. Mov., 26:77-92, 2011.

Vernetta Santana, M.; Jiménez Rodríguez, J. \& López Bedoya, J. La utilización del registro de los tiempos de intervención de las acciones motrices en la gimnasia acrobática. E. F. Deportes.com Rev. Digit., 12(110), 2007.

\author{
Dirección para Correspondencia: \\ Yaiza Taboada Iglesias \\ Departamento de Didácticas Especiales \\ Facultad de Ciencias de la Educación y del Deporte \\ Universidad de Vigo \\ Campus A Xunqueira s/n 36005 \\ Pontevedra \\ ESPAÑA
}

Tel: 986802054

Fax: 986801701

Email: yaitaboada@uvigo.es

Recibido: 17-12-2014

Aceptado: 28-05-2015 